\title{
Equity value, bankruptcy, and optimal dividend policy with finite maturity - variational inequality approach with discontinuous coefficient
}

Xiaoru $\operatorname{Han}^{1,2^{*}}$ and Fahuai Yi ${ }^{2}$

Correspondence:

13424608805@126.com

1 Department of Mathematics,

Foshan University, Guangdong, 528000, China

${ }^{2}$ School of Mathematical Sciences, South China Normal University,

Guangzhou, China

\begin{abstract}
This article examines the value of equity, optimal bankruptcy boundary, and optimal dividend policy in a continuous-time framework with finite time maturity. The model of equity value is formulated as a parabolic variational inequality, or equivalently, a free boundary problem, where the free boundary corresponds to the optimal bankruptcy boundary. We present an analytical approach to analyze the behaviors of the free boundary. The regularity of the value function and the optimal dividend policy are studied as well. The main feature and difficulty are the discontinuity of the coefficient in the variational inequality.
\end{abstract}

MSC: 35R35; 47J20; 93E20

Keywords: equity value; optimal bankruptcy boundary; finite horizon; optimal dividend policy

\section{Introduction}

The value of debt and equity and default covenants have long been interested in corporate finance literature. Merton [1] pioneers the study of corporate debt and equity value under the assumption that bankruptcy cannot be triggered before maturity, he obtained the value of debt and equity. Black and Cox [2], Leland [3], Anderson and Sundaresan [4], Bruche [5] and Zhou [6] have extended the original Merton [1] model to incorporate a more realistic assumption: the possibility of early default. By assuming a time-independent debt structure, Leland [3] derived closed-form solutions for the value of debt and for optimal capital structure.

Unfortunately in these models dividend policy is not explicitly considered. The firms either simply do not have a policy with respect to dividends (Leland [3]) or they pay out all residual cash flows as dividends (Anderson and Sundaresan [4], Leland and Toft [7]). Fan and Sundaresan [8] and Gryglewicz [9] study the optimal dividend policy. In [8], dividends are treated as a control variable in the firm's cash flow generating process. They obtained the optimal dividend policy. Gryglewicz [9] presented a model of a firm that optimally chooses capital structure, cash holding, dividends, and default. However, most of these papers were restricted to dealing with a perpetual debt.

(c) 2015 Han and Yi; licensee Springer. This is an Open Access article distributed under the terms of the Creative Commons Attribution License (http://creativecommons.org/licenses/by/4.0), which permits unrestricted use, distribution, and reproduction in any medium, provided the original work is properly credited. 
It is challenging to take the finite horizon case into consideration since the debt values are no longer time homogeneous. The fundamental valuation equation will explicitly depend on time.

In this paper, we aim to develop a continuous-time model for the finite horizon corporate equity, provide a theoretical analysis of the behaviors of the optimal bankruptcy boundary, and obtain the optimal dividend policy. One paper related to the present work is Han et al. [10] where they obtained the optimal reorganization boundary by using a variational inequality approach, but they did not consider the dividend policy; they paid out all residual cash flows as dividends. However, in our formulation, dividend is optional when cash payout exceeds the promised coupon rate. That is to say, when $\beta V \geq c$, stockholders have a decision to make: they can pay all the residual cash flows as dividends to themselves, or they can reinvest a fraction to the firm. They choose their dividend policy by acting to maximize their equity value. Whenever $\beta V<c$, the firm is under a liquidity constraint and no dividend can be paid (see (2.2) and (2.4)), which causes the function $f(v)$ (in (2.6)) to be not continuous at the point $V=c / \beta$ and the inequality $\partial_{x} u \geq 0$ does not hold. But the condition $\partial_{x} u \geq 0$ is critical to prove the smoothness and monotonicity of the free boundary. At this point, it brings difficulty for analyzing the behaviors of the free boundary.

The rest of the paper is organized as follows. In Section 2, we set up the model. In Section 3, we study the behavior of the solution and prove that when $\beta V \geq c$, as the payout ratio increases from $c(1-\gamma) / V$ to $\beta$, the firm's equity value is increasing, in other words, it would be optimal for the equity holders to pay all the cash flows available as dividends. Section 4 is devoted to an analysis of the behaviors of the free boundary in case of $c(1-\gamma)-r P<0$, we prove that the free boundary is decreasing and infinitely differentiable. In Section 5, we deduce the boundedness of the free boundary by the comparison principle and show that it is not always monotonic in some case. In Section 6 we provide some numerical results and some financial interpretations. We conclude in Section 7. The main contribution of this paper lies in the following. 1. We give a rigorous derivation of variational inequality (2.6) by stochastic analysis (Section 2 ). 2 . We prove that the value of equity is increasing with the aggregate payout ratio $\delta$ and obtaining the optimal dividend policy (Section 3). 3. In the absence of $\partial_{x} u \geq 0$, we show the monotonicity and infinite differentiability of the bankruptcy boundary for the case of $c(1-\gamma)-r P \leq 0$ (Section 4). 4. We analyze the loss of monotonicity of the reorganization boundary in some cases for the case of $c(1-\gamma)-r P>0$ (Section 5). 5. We give some numerical results and financial interpretations (Section 6). The main feature and difficulty are the discontinuity of the coefficient $f(v)$ in variational inequality (2.6), which brings about a lot of trouble.

\section{Formulation of the model}

In this section, we develop a model of equity value with finite time maturity at time $T$. The model is set in a continuous-time framework. The following assumptions underlie the model:

(1) There is a firm which has equity and a single issue of debt which promises a flow rate of coupon $c$ per unit time. The principal amount of the debt is $P$.

(2) To focus attention on default risk, we assume that the default-free term structure is flat and the instantaneous risk-free rate is $r$ per unit time.

(3) When the firm pays its contractual coupon $c$, it is entitled to a tax benefit of $\gamma c$ $(0 \leq \gamma \leq 1)$. During the default period, the tax benefits are lost.

(4) Asset sales for dividend payments are prohibited. 
(5) The firm is not otherwise constrained by covenants, bankruptcy will occur only when the firm cannot meet the required (instantaneous) coupon payment: that is, when the equity value falls to zero. In fact, in continuous time, the coupon $(c d t)$ paid over the infinitesimal interval, $d t$, is itself infinitesimal. Therefore the value of equity simply needs to be positive to avoid bankruptcy over the next instant.

(6) The asset value of the firm, denoted by $V$, follows the lognormal diffusion process,

$$
d V=(\mu-\beta) V d t+\sigma V d B_{t}
$$

where $\mu$ is the instantaneous expected rate of return on the firm gross of all payout, $\sigma^{2}$ is the instantaneous variance of the return on the firm, $B_{t}$ is the standard Brownian motion. The cash payout at any time is $\beta V$ and $\beta \leq \mu$, where $\beta$ is the firm's cash payout ratio and the free cash flows available for the payment of dividends and debt service is restrict to $\beta V$.

Most models in the literature tend to assume that the residual cash flow are simply paid out as dividends. In our formulation, as Fan and Sundaresan in [8], dividends, or equivalently the total payout ratio, denoted by $\delta$, are treated as a control variable in the firm's cash flow generating process. Stockholders will choose their dividend policies by acting to maximize their equity value.

When the cash payout $\beta V$ exceeds the promised coupon rate $c$, stockholders have a decision to make: they can pay all the residual cash flows as dividends to themselves, or they can reinvest a fraction into the firm. The motivation for such an action is simple: by foregoing current dividends, the stockholders can avoid costly liquidations that may arise in the future. This feature is modeled in the following manner.

Whenever $\beta V \geq c$, the firm has no flow constraint and we refer to this state as a 'good' state. We assume that the dynamics of the firm's value is given by

$$
d V=(\mu-\beta) V d t+\sigma V d B_{t}+(\beta-\delta) V d t=(\mu-\delta) V d t+\sigma V d B_{t}
$$

where $\delta$ denotes the aggregate payout ratio. Among the total cash flow $\beta V$, the retained earnings, $(\beta-\delta) V$, are reinvested back into the firm's value-generating activity. The total payout $\delta V$ includes the coupon payment $c$ and the dividends $\delta V-c$. When $\beta V \geq c$, we constrain

$$
c(1-\gamma) / V \leq \delta \leq \beta
$$

since the payout at least has to cover the debt obligations and no more than the total cash flows being available.

Whenever $\beta V<c$, the firm is under a liquidity constraint and we refer to this state as a 'bad' state. We assume that the dynamics of the firm's value is given by

$$
d V=(\mu-\beta) V d t+\sigma V d B_{t}
$$

No dividend can be paid because equity holders are not allowed to pay themselves dividend by selling the firm's assets. In order to address this issue systematically, we begin with a given payout ratio $\delta$.

Definition Let $0 \leq t \leq T, V(t)=v \geq 0, \mathcal{F}_{u}^{(t)}(t \leq u \leq T)$ be the filtration of $V(t)$, $\mathcal{T}_{t, T}$ be the set of all stopping time in $[t, T]$. The value of equity with maturity time $T$ at time $t$ is 
defined as

$$
\begin{aligned}
E(v, t)= & \max _{\tau \in \mathcal{T}_{t, T}} \widetilde{E}\left[e^{-r(\tau-t)}(V(\tau)-P)^{+} \mathcal{X}_{\{\tau=T\}}-\int_{t}^{\tau} e^{-r(u-t)} c(1-\gamma) d u\right. \\
& \left.+\int_{t}^{\tau} e^{-r(u-t)}\left(\beta \mathcal{X}_{\left\{V_{u}<c \mid \beta\right\}}+\delta \mathcal{X}_{\left\{V_{u} \geq c / \beta\right\}}\right) V_{u} d u \mid V(t)=v\right],
\end{aligned}
$$

where $\widetilde{E}$ is the expectation under the risk neutral measure, $\mathcal{X}_{A}$ denotes the indicator function of the set $A$, the second term $\widetilde{E}\left[\int_{t}^{\tau} e^{-r(u-t)} c(1-\gamma) d u\right]$ denotes the discounted expected value of coupon interest, the third term $\widetilde{E}\left[\int_{t}^{\tau} e^{-r(u-t)}\left(\beta \mathcal{X}_{\left\{V_{u}<c / \beta\right\}}+\delta \mathcal{X}_{\left\{V_{u} \geq c / \beta\right\}}\right) V_{u} d u\right]$ represents the expected discounted value of cash payout for dividends and debt service.

Theorem 2.1 The value of equity $E(v, t)$ satisfies the following parabolic variational inequality:

$$
\begin{cases}E(v, t) \geq 0, & v>0,0 \leq t<T, \\ -\partial_{t} E-\frac{1}{2} \sigma^{2} v^{2} \partial_{v v} E-(r v-f(v)) \partial_{v} E+r E-f(v)+c(1-\gamma) \geq 0, & v>0,0<t<T, \\ E\left[-\partial_{t} E-\frac{1}{2} \sigma^{2} v^{2} \partial_{v v} E-(r v-f(v)) \partial_{v} E+r E-f(v)+c(1-\gamma)\right]=0, & v>0,0<t<T, \\ E(v, T)=(v-P)^{+}, & v>0,\end{cases}
$$

where

$$
f(v)= \begin{cases}\beta v, & v \leq c / \beta, \\ \delta v, & v>c / \beta .\end{cases}
$$

Proof From the definition of $E(v, t)$, we set $\tau=t$ and $t=T$ in (2.5), respectively, it is clear that

$$
E(v, t) \geq 0, \quad E(v, T)=(v-P)^{+} .
$$

Let us consider the time $\tau=t+h(h>0)$, by the dynamic programming principle [11],

$$
\begin{aligned}
& \widetilde{E}\left\{e^{-r(t+h)} E\left(V_{t+h}, t+h\right)+\int_{t}^{t+h} e^{-r u}\left[\left(\beta \mathcal{X}_{\left\{V_{u}<c / \beta\right\}}+\delta \mathcal{X}_{\left\{V_{u} \geq c / \beta\right\}}\right) V_{u}-c(1-\gamma)\right] d u\right\} \\
& \quad \leq e^{-r t} E(v, t),
\end{aligned}
$$

i.e.

$$
\widetilde{E}\left\{\int_{t}^{t+h} d\left[e^{-r u} E\left(V_{u}, u\right)\right]+\int_{t}^{t+h} e^{-r u}\left[\left(\beta \mathcal{X}_{\left\{V_{u}<c \mid \beta\right\}}+\delta \mathcal{X}_{\left\{V_{u} \geq c / \beta\right\}}\right) V_{u}-c(1-\gamma)\right] d u\right\}
$$$$
\leq 0 \text {. }
$$

From (2.2) and (2.4), we can get

$$
d V=\left(r-\beta \mathcal{X}_{\{V<c / \beta\}}-\delta \mathcal{X}_{\{V \geq c / \beta\}}\right) V d t+\sigma V d \widetilde{B}_{t},
$$

where $\widetilde{B}_{t}$ is a standard Brownian motion under the risk neutral measure $\widetilde{P}$. 
We apply the Itô formula:

$$
\begin{aligned}
& d\left[e^{-r u} E\left(V_{u}, u\right)\right] \\
& =e^{-r u}\left[\partial_{t} E\left(V_{u}, u\right)+\frac{1}{2} \sigma^{2}\left(V_{u}\right)^{2} \partial_{v v} E\left(V_{u}, u\right)-r E\left(V_{u}, u\right)\right. \\
& \left.\quad+\left(r-\beta \mathcal{X}_{\left\{V_{u}<c / \beta\right\}}-\delta \mathcal{X}_{\left\{V_{u} \geq c / \beta\right\}}\right) V_{u} \partial_{v} E\left(V_{u}, u\right)\right] d u+e^{-r u} \sigma V_{u} \partial_{v} E\left(V_{u}, u\right) d \widetilde{B}_{t} .
\end{aligned}
$$

Substituting (2.9) into (2.7), dividing by $h$ and sending $h$ to 0 , this yield by the mean-value theorem

$$
-\partial_{t} E-\frac{1}{2} \sigma^{2} v^{2} \partial_{\nu v} E-(r v-f(v)) \partial_{v} E+r E-f(v)+c(1-\gamma) \geq 0 .
$$

When $E(v, t)>0$, which implies the optimal stopping time $\tau^{*}>t$, then we set $\tau=\tau^{*}$, from the dynamic programming principle,

$$
e^{-r t} E(v, t)=\widetilde{E}\left\{e^{-r \tau^{*}} E\left(V_{\tau^{*}}, \tau^{*}\right)+\int_{t}^{\tau^{*}} e^{-r u}\left[\left(\beta \mathcal{X}_{\left\{V_{u}<c / \beta\right\}}+\delta \mathcal{X}_{\left\{V_{u} \geq c / \beta\right\}}\right) V_{u}-c(1-\gamma)\right] d u\right\},
$$

i.e.

$$
\widetilde{E}\left\{\int_{t}^{\tau^{*}} d\left[e^{-r u} E\left(V_{u}, u\right)\right]+\int_{t}^{\tau^{*}} e^{-r u}\left[\left(\beta \mathcal{X}_{\left\{V_{u}<c / \beta\right\}}+\delta \mathcal{X}_{\left\{V_{u} \geq c / \beta\right\}}\right) V_{u}-c(1-\gamma)\right] d u\right\}=0 .
$$

Combining with (2.9) and noting that $\tau^{*}>t$, we obtain

$$
-\partial_{t} E-\frac{1}{2} \sigma^{2} v^{2} \partial_{v v} E-(r v-f(v)) \partial_{v} E+r E-f(v)+c(1-\gamma)=0 .
$$

In view of problem (2.6) being a backward PDE and the degeneracy of the operator $\mathcal{L}_{v}$ at $v=0$, we set

$$
x=\ln v, \quad \tau=T-t, \quad u(x, \tau)=E(v, t),
$$

then we have

$$
\begin{cases}u \geq 0, & (x, \tau) \in \Omega_{T}, \\ \partial_{\tau} u-\mathcal{L} u+g(x)\left(\partial_{x} u-e^{x}\right)+c(1-\gamma) \geq 0, & (x, \tau) \in \Omega_{T}, \\ u\left[\partial_{\tau} u-\mathcal{L} u+g(x)\left(\partial_{x} u-e^{x}\right)+c(1-\gamma)\right]=0, & (x, \tau) \in \Omega_{T}, \\ u(x, 0)=\left(e^{x}-P\right)^{+}, & x \in R,\end{cases}
$$

where $\Omega_{T}=R \times(0, T]$ and

$$
g(x)= \begin{cases}\beta, & x \leq \ln \frac{c}{\beta}, \\ \delta, & x>\ln \frac{c}{\beta},\end{cases}
$$

and

$$
\mathcal{L} u=\frac{1}{2} \sigma^{2} \partial_{x x} u+\left(r-\frac{1}{2} \sigma^{2}\right) \partial_{x} u-r u .
$$




\section{The behavior of solution of problem (2.10)}

Since problem (2.10) lies in an unbounded region and $g(x)$ is not smooth, we first consider the problem in the bounded domain $\Omega_{T}^{n}=(-n, n) \times(0, T], n \in \mathbb{N} \backslash\{0\}$ :

$$
\begin{cases}u_{n} \geq 0, & (x, \tau) \in \Omega_{T}^{n}, \\ \partial_{\tau} u_{n}-\mathcal{L} u_{n}+g_{n}(x)\left(\partial_{x} u_{n}-e^{x}\right)+c(1-\gamma) \geq 0, & (x, \tau) \in \Omega_{T}^{n}, \\ u_{n}\left[\partial_{\tau} u_{n}-\mathcal{L} u_{n}+g_{n}(x)\left(\partial_{x} u_{n}-e^{x}\right)+c(1-\gamma)\right]=0, & (x, \tau) \in \Omega_{T}^{n}, \\ \partial_{x} u_{n}(-n, \tau)-u_{n}(-n, \tau)=0, & \tau \in(0, T], \\ \partial_{x} u_{n}(n, \tau)=e^{n}, & \tau \in(0, T], \\ u_{n}(x, 0)=\left(e^{x}-P\right)^{+} & x \in[-n, n],\end{cases}
$$

where $g_{n}(x)$ satisfies

$$
g_{n}(x) \in C^{1}[-n, n], \quad \delta \leq g_{n}(x) \leq \beta, \quad \text { for all } x \in[-n, n]
$$

moreover,

$$
g_{n}(x)= \begin{cases}\beta, & x<\ln \frac{c}{\beta}-\frac{1}{n}, \\ \text { decreasing, } & \ln \frac{c}{\beta}-\frac{1}{n}<x<\ln \frac{c}{\beta}+\frac{1}{n}, \\ \delta, & x \geq \ln \frac{c}{\beta}+\frac{1}{n} .\end{cases}
$$

Lemma 3.1 For any fixed $n \in \mathbb{N} \backslash\{0\}$, there exists a solution $u_{n} \in C\left(\overline{\Omega_{T}^{n}}\right) \cap W_{p}^{2,1}\left(\Omega_{T}^{n} \backslash B_{\theta}\left(P_{0}\right)\right)$ to problem (3.1), where $1<p<\infty, \theta>0 ; P_{0}=(\ln P, 0), B_{\theta}\left(P_{0}\right)=\left\{(x, \tau):(x-\ln P)^{2}+\tau^{2} \leq \theta^{2}\right\}$. Moreover, if $n$ is large enough, we have

$$
0 \leq u_{n} \leq e^{x}, \quad(x, \tau) \in \Omega_{T}^{n}
$$

Proof As usual [12], we define a penalty function $\beta_{\varepsilon}(t)$ (see Figure 1), which satisfies

$$
\begin{aligned}
& \beta_{\varepsilon}(t) \in C^{2}(-\infty,+\infty), \quad \beta_{\varepsilon}(t) \leq 0 \text { for all } t \in R, \\
& \beta_{\varepsilon}(t)=0 \quad \text { if } t \geq \varepsilon, \quad \beta_{\varepsilon}(0)=-c(1-\gamma), \\
& \beta_{\varepsilon}^{\prime}(t) \geq 0, \quad \beta_{\varepsilon}^{\prime \prime}(t) \leq 0,
\end{aligned}
$$

moreover,

$$
\lim _{\varepsilon \rightarrow 0^{+}} \beta_{\varepsilon}(t)= \begin{cases}0, & t>0 \\ -\infty, & t<0 .\end{cases}
$$

Since $\left(e^{x}-P\right)^{+}$is not in $W_{p}^{2}([-n, n])$, we need to smooth it. Define $\pi_{\varepsilon}(t)$ (see Figure 2) as

$$
\pi_{\varepsilon}(t)= \begin{cases}t, & t \geq \varepsilon \\ 0, & t \leq-\varepsilon\end{cases}
$$

and $\pi_{\varepsilon}(t) \in C^{\infty}, 0 \leq \pi_{\varepsilon}^{\prime}(t) \leq 1, \pi_{\varepsilon}^{\prime \prime}(t) \geq 0, \lim _{\varepsilon \rightarrow 0^{+}} \pi_{\varepsilon}(t)=t^{+}$. 
Figure $1 \beta_{\varepsilon}(t)$.

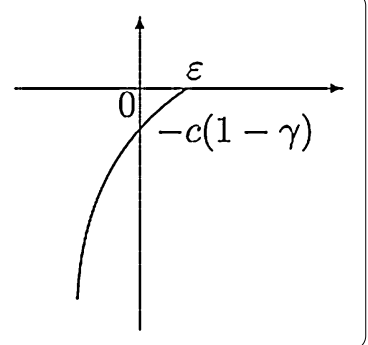

Figure $2 \pi_{\varepsilon}(t)$.

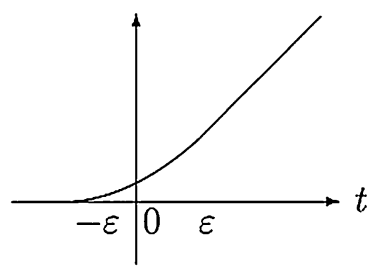

Following the idea in [12], we construct an approximation of problem (3.1)

$$
\begin{cases}\partial_{\tau} u_{\varepsilon, n}-\mathcal{L} u_{\varepsilon, n}+g_{n}(x)\left(\partial_{x} u_{\varepsilon, n}-e^{x}\right)+\beta_{\varepsilon}\left(u_{\varepsilon, n}\right)+c(1-\gamma)=0, & (x, \tau) \in \Omega_{T}^{n}, \\ \partial_{x} u_{\varepsilon, n}(-n, \tau)-u_{\varepsilon, n}(-n, \tau)=0, & \tau \in(0, T], \\ \partial_{x} u_{\varepsilon, n}(n, \tau)=e^{n}, & \tau \in(0, T], \\ u_{\varepsilon, n}(x, 0)=\pi_{\varepsilon}\left(e^{x}-P\right), & x \in[-n, n] .\end{cases}
$$

By applying the Schauder fixed theorem we can establish the existence of the $W_{p}^{2,1}$ solution to problem (3.3). The procedure is standard, we omit the details.

If we can prove that as $n$ is large enough

$$
0 \leq u_{\varepsilon, n} \leq e^{x}
$$

then by the method in [12], it is not difficult to deduce that, as $\varepsilon \rightarrow 0^{+}$,

$$
u_{\varepsilon, n} \rightarrow u_{n} \quad \text { in } W_{p}^{2,1}\left(\Omega_{T}^{n} \backslash B_{\theta}\left(P_{0}\right)\right) \text { weakly, and } \quad u_{\varepsilon, n} \rightarrow u_{n} \quad \text { in } C\left(\overline{\Omega_{T}^{n}}\right)
$$

where $u_{n}$ is the solution of problem (3.1).

Next, we prove (3.4). We set $w_{1}=0$, then

$$
\begin{aligned}
\partial_{\tau} w_{1}-\mathcal{L} w_{1}+g_{n}(x)\left(\partial_{x} w_{1}-e^{x}\right)+\beta_{\varepsilon}\left(w_{1}\right)+c(1-\gamma) \\
\quad=-g_{n}(x) e^{x}+\beta_{\varepsilon}(0)+c(1-\gamma) \\
\quad=-g_{n}(x) e^{x} \leq 0
\end{aligned}
$$

(The last equity is from the definition of $\beta_{\varepsilon}(0)$.) 
Moreover, from the boundary conditions in (3.3), we see that

$$
\begin{cases}\partial_{x} u_{\varepsilon, n}(-n, \tau)-u_{\varepsilon, n}(-n, \tau)=0=\partial_{x} w_{1}(-n, \tau)-w_{1}(-n, \tau), & \tau \in(0, T], \\ \partial_{x} u_{\varepsilon, n}(n, \tau)=e^{n} \geq \partial_{x} w_{1}(n, \tau), & \tau \in(0, T], \\ u_{\varepsilon, n}(x, 0)=\pi_{\varepsilon}\left(e^{x}-P\right) \geq w_{1}(x, 0), & x \in[-n, n] .\end{cases}
$$

Applying the comparison principle of PDE [13], we have $u_{\varepsilon, n} \geq 0$, that is, the left hand side of inequality (3.4). On the other hand, we set $w_{2}=e^{x}$, then

$$
\begin{aligned}
\partial_{\tau} w_{2}-\mathcal{L} w_{2}+g_{n}(x)\left(\partial_{x} w_{2}-e^{x}\right)+\beta_{\varepsilon}\left(w_{2}\right)+c(1-\gamma) \\
=\beta_{\varepsilon}\left(e^{x}\right)+c(1-\gamma) \\
=c(1-\gamma) \geq 0 \quad\left(\text { for any } 0<\varepsilon<e^{-n}\right),
\end{aligned}
$$

and from the boundary condition in (3.3), we have

$$
\begin{cases}\partial_{x} u_{\varepsilon, n}(-n, \tau)-u_{\varepsilon, n}(-n, \tau)=0=\partial_{x} w_{2}(-n, \tau)-w_{2}(-n, \tau), & \tau \in(0, T] \\ \partial_{x} u_{\varepsilon, n}(n, \tau)=e^{n}=\partial_{x} w_{2}(n, \tau), & \tau \in(0, T] \\ u_{\varepsilon, n}(x, 0)=\pi_{\varepsilon}\left(e^{x}-P\right) \leq e^{x}=w_{2}(x, 0) . & \end{cases}
$$

Combining (3.3), applying the comparison principle of PDE [13], we have $u_{\varepsilon, n} \leq w_{2}$. Hence, we obtain (3.4), and (3.2) is the consequence of (3.4).

Lemma 3.2 For problem (3.1), the solution $u_{n}(x, \tau)$ satisfies

$$
\partial_{x} u_{n} \leq e^{x}, \quad(x, \tau) \in \Omega_{T}^{n} .
$$

Proof Denote $\partial_{x} u_{\varepsilon, n}=w_{3}$. Differentiating the equation in (3.3) with respect to $x$,

$$
\partial_{\tau} w_{3}-\mathcal{L} w_{3}+g_{n}(x) \partial_{x}\left(w_{3}-e^{x}\right)+g_{n}^{\prime}(x)\left(w_{3}-e^{x}\right)+\beta_{\varepsilon}^{\prime}\left(u_{\varepsilon, n}\right) w_{3}=0, \quad(x, \tau) \in \Omega_{T}^{n},
$$

it can be rewritten as

$$
\begin{aligned}
& \partial_{\tau}\left(w_{3}-e^{x}\right)-\mathcal{L}\left(w_{3}-e^{x}\right)+g_{n}(x) \partial_{x}\left(w_{3}-e^{x}\right)+g_{n}^{\prime}(x)\left(w_{3}-e^{x}\right)+\beta_{\varepsilon}^{\prime}\left(u_{\varepsilon, n}\right)\left(w_{3}-e^{x}\right) \\
& \quad=-\beta_{\varepsilon}^{\prime}\left(u_{\varepsilon, n}\right) e^{x} \leq 0, \quad(x, \tau) \in \Omega_{T}^{n} .
\end{aligned}
$$

From $(3.3)_{2}$,

$$
\partial_{x} u_{\varepsilon, n}(-n, \tau)=u_{\varepsilon, n}(-n, \tau)
$$

it follows that, by (3.4), $0 \leq w_{3}(-n, \tau) \leq e^{-n}$,

$$
w_{3}(-n, \tau)-e^{-n} \leq 0 .
$$

It is clear that, by $(3.3)_{3,4}$,

$$
w_{3}(n, \tau)-e^{n}=0, \quad w_{3}(x, 0)-e^{x} \leq 0 .
$$


Hence

$$
\partial_{x} u_{n} \leq e^{x}
$$

Theorem 3.1 There exists a solution $u \in C\left(\bar{\Omega}_{T}\right) \cap W_{p}^{2,1}\left(\Omega_{T}^{R} \backslash B_{\delta}\left(P_{0}\right)\right)$ to problem (2.10) for any $1<p<\infty, \delta>0, R>0$, and

$$
\begin{aligned}
& 0 \leq u \leq e^{x}, \quad(x, \tau) \in \Omega_{T} \\
& \partial_{x} u \leq e^{x}, \quad(x, \tau) \in \Omega_{T},
\end{aligned}
$$

moreover, the solution satisfying (3.6) is unique, and

$$
u>0, \quad(x, \tau) \in\left\{(x, \tau): x>\ln \frac{c}{\beta}, 0<\tau<T\right\} \triangleq Q_{T} .
$$

Proof We rewrite problem (3.1) as

$$
\begin{cases}\partial_{\tau} u_{n}-\mathcal{L} u_{n}+g_{n}(x) \partial_{x} u_{n}=F(x, \tau), & \text { in } \Omega_{T}^{n}, \\ \partial_{x} u_{n}(-n, \tau)-u_{n}(-n, \tau)=0, & \tau \in(0, T], \\ \partial_{x} u_{n}(n, \tau)=e^{n}, & \tau \in(0, T], \\ u_{n}(x, 0)=\left(e^{x}-P\right)^{+}, & x \in[-n, n],\end{cases}
$$

$u_{n} \in W_{p, \text { loc }}^{2,1}\left(\Omega_{T}^{n}\right)$ implies $F(x, \tau) \in L_{\mathrm{loc}}^{p}\left(\Omega_{T}^{n}\right)$ where

$$
F(x, \tau)=\left(g_{n}(x) e^{x}-c(1-\gamma)\right) \mathcal{X}_{\left\{u_{n}>0\right\}}, \quad \text { a.e. } \Omega_{T}^{n} \text {. }
$$

As $\delta \leq g_{n}(x) \leq \beta$, it is obvious that $|F(x, \tau)| \leq C$ for $-R \leq x \leq R$, where $R>0, C$ is dependent on $R$, but independent of $n$. Hence, for any fixed $R>0,0<\theta<\min \{|R-\ln P|,|R+\ln P|\}$, if $n>R$, combining (3.2), we have the following $W_{p}^{2,1}$ uniform estimates in the domain $\Omega_{T}^{R} \backslash B_{\theta}\left(P_{0}\right):$

$$
\begin{aligned}
& \left|u_{n}\right|_{W_{p}^{2,1}\left(\Omega_{T}^{R} \backslash B_{\theta}\left(P_{0}\right)\right)} \\
& \quad \leq C\left[\left|u_{n}\right|_{L^{\infty}\left(\Omega_{T}^{R}\right)}+|F(x, \tau)|_{L^{\infty}\left(\Omega_{T}^{R}\right)}+\left|e^{x}-P\right|_{C^{2}([-R,-\theta+\ln P] \cup[\theta+\ln P, R])}\right] \\
& \quad \leq C
\end{aligned}
$$

where $C$ depends on $R, \theta$, but is independent of $n$. Let $n \rightarrow \infty$, then we have possibly a subsequence,

$$
\begin{aligned}
& u_{n} \rightarrow u^{R} \quad \text { in } W_{p}^{2,1}\left(\Omega_{T}^{R} \backslash B_{\theta}\left(P_{0}\right)\right) \text { weakly as } n \rightarrow \infty, \\
& \partial_{x} u_{n} \rightarrow \partial_{x} u^{R} \quad \text { in } C\left(\Omega_{T}^{R} \backslash B_{\theta}\left(P_{0}\right)\right) \text { as } n \rightarrow \infty .
\end{aligned}
$$

Applying a $C^{\alpha}$ estimate (see [14] and [13]) we have

$$
\left|u_{n}\right|_{C^{\alpha, \frac{\alpha}{2}}}\left(\overline{\Omega_{T}^{R}}\right) \leq C
$$


where $C$ depends on $R$, but is independent of $n$. It follows that

$$
u_{n} \rightarrow u^{R} \quad \text { in } C\left(\bar{\Omega}_{T}^{R}\right) \text { as } n \rightarrow \infty .
$$

Define $u=u^{R}$ if $x \in[-R, R]$ for each $R>0$, it is clear that $u$ is reasonable defined in $\bar{\Omega}_{T}$ and $u \in C\left(\overline{\Omega_{T}}\right) \cap W_{p}^{2,1}\left(\Omega_{T}^{R} \backslash B_{\delta}\left(P_{0}\right)\right)$ is the solution of problem (2.10). Equations (3.6) and (3.7) are consequences of (3.2) and (3.5).

Next, we prove uniqueness. Suppose $U_{1}$ and $U_{2}$ are two $C\left(\overline{\Omega_{T}}\right) \cap W_{p, \text { loc }}^{2,1}\left(\Omega_{T}^{R}\right)$ solutions to problem (2.10) and satisfy (3.6); denote

$$
\mathcal{N}=\left\{(x, \tau): U_{1}(x, \tau)<U_{2}(x, \tau), x \in R, 0<\tau \leq T\right\}
$$

and suppose it is not empty. Then if $(x, \tau) \in \mathcal{N}$,

$$
U_{2}(x, \tau)>0, \quad \partial_{\tau} U_{2}-\mathcal{L} U_{2}+g(x) \partial_{x} U_{2}=g(x) e^{x}-c(1-\gamma) .
$$

Denote $W=U_{2}-U_{1}$, then $W$ satisfies

$$
\begin{cases}\partial_{\tau} W-\mathcal{L} W+g(x) \partial_{x} W \leq 0, & (x, \tau) \in \mathcal{N}, \\ W(x, \tau)=0, & (x, \tau) \in \partial_{p} \mathcal{N}\end{cases}
$$

where $\partial_{p} \mathcal{N}$ is the parabolic boundary of the domain $\mathcal{N}$. From (3.6), we know the function $W$ satisfies the estimation $0 \leq W \leq e^{x}$, so using the maximum principle [15], we deduce $W \leq 0$ in $\mathcal{N}$, which contradicts the definition of $\mathcal{N}$.

Finally, we prove the estimate (3.8). From problem (2.10), we see that

$$
\begin{cases}\partial_{\tau} u-\frac{1}{2} \sigma^{2} \partial_{x x} u-\left(r-\delta-\frac{1}{2} \sigma^{2}\right) \partial_{x} u+r u \geq \delta e^{x}-c(1-\gamma) \geq 0, & (x, \tau) \in Q_{T}, \\ u\left(\ln \frac{c}{\beta}, \tau\right) \geq 0, & \tau \in(0, T), \\ u(x, 0)=\left(e^{x}-P\right)^{+} \geq 0, & x>\ln \frac{c}{\beta} .\end{cases}
$$

In fact, the inequality in (3.9) is deduced from (2.3). Using the strong maximum principle, we deduce that $u>0$ in $Q_{T}$.

Theorem 3.2 The solution of problem (2.10) is increasing with the aggregate payout ratio $\delta$, it is to say that when $\beta e^{x} \geq c$, as the payout ratio $\delta$ increases from $c(1-\gamma) e^{-x}$ to $\beta$, the firm's equity value is increasing. Thus we conclude that when $\beta V \geq c$, it is optimal to pay all the residual cash flows as dividends. When $\beta V<c$, it is optimal to pay no dividends.

Proof Denote that $u_{\epsilon, n}^{i}(i=1,2)$ is the solution of problem (3.3) (where $\delta$ is replaced by $\delta^{i}$ )

$$
\begin{cases}\partial_{\tau} u_{\varepsilon, n}^{i}-\mathcal{L} u_{\varepsilon, n}^{i}+g_{n}^{i}(x)\left(\partial_{x} u_{\varepsilon, n}^{i}-e^{x}\right)+\beta_{\varepsilon}\left(u_{\varepsilon, n}^{i}\right)+c(1-\gamma)=0, & (x, \tau) \in \Omega_{T}^{n}, \\ \partial_{x} u_{\varepsilon, n}^{i}(-n, \tau)-u_{\varepsilon, n}^{i}(-n, \tau)=0, & \tau \in(0, T], \\ \partial_{x} u_{\varepsilon, n}^{i}(n, \tau)=e^{n}, & \tau \in(0, T], \\ u_{\varepsilon, n}^{i}(x, 0)=\pi_{\varepsilon}\left(e^{x}-P\right), & x \in[-n, n],\end{cases}
$$


where $g_{n}^{i}(x)$ satisfies

$$
g_{n}^{i}(x)= \begin{cases}\beta, & x<\ln \frac{c}{\beta}-\frac{1}{n} \\ \text { decreasing, } & \ln \frac{c}{\beta}-\frac{1}{n}<x<\ln \frac{c}{\beta}+\frac{1}{n} \\ \delta^{i}, & x \geq \ln \frac{c}{\beta}+\frac{1}{n}\end{cases}
$$

moreover,

$$
g_{n}^{i}(x) \in C^{1}[-n, n], \quad \delta^{i} \leq g_{n}^{i}(x) \leq \beta, \quad \text { for all } x \in[-n, n],
$$

and $g_{n}^{1}(x) \leq g_{n}^{2}(x)$ if $\delta^{1} \leq \delta^{2}$.

We assume that $\delta^{1}<\delta^{2}$, denote $w=u_{\epsilon, n}^{1}-u_{\epsilon, n}^{2}$, then $w$ satisfies

$$
\begin{cases}\partial_{\tau} w-\mathcal{L} w+g_{n}^{1}(x) \partial_{x} w+\beta_{\varepsilon}^{\prime}(\cdot) w=-\left(g_{n}^{1}(x)-g_{n}^{2}(x)\right)\left(\partial_{x} u_{\varepsilon, n}^{2}-e^{x}\right), & (x, \tau) \in \Omega_{T}^{n}, \\ \partial_{x} w(-n, \tau)-w(-n, \tau)=0, & \tau \in(0, T], \\ \partial_{x} w(n, \tau)=0, & \tau \in(0, T], \\ w(x, 0)=0, & x \in[-n, n] .\end{cases}
$$

In fact, from the estimation (3.5), $-\left(g_{n}^{1}(x)-g_{n}^{2}(x)\right)\left(\partial_{x} u_{\varepsilon, n}^{2}-e^{x}\right) \leq 0$. By the maximum principle, we deduce that $w \leq 0$, i.e. $u_{\epsilon, n}^{1} \leq u_{\epsilon, n}^{2}$. Let $\epsilon \rightarrow 0$ and $n \rightarrow \infty$, we obtain

$$
u^{1}(x, \tau) \leq u^{2}(x, \tau), \quad(x, \tau) \in \Omega_{T}
$$

where $u^{i}(x, \tau)(i=1,2)$ is the solution of problem (2.10) with the payout ratio $\delta^{i}$.

Remark 3.1 Theorem 3.2 shows that, when $\beta e^{x} \geq c$, as the payout ratio $\delta$ increases from $c(1-\gamma) e^{-x}$ to $\beta$, the firm's equity value is increasing, thus we obtain

$$
u(x, \tau) \leq u^{\beta}(x, \tau), \quad(x, \tau) \in \Omega_{T}
$$

where $u^{\beta}(x, \tau)$ is the solution of problem (2.10) with $\delta=\beta$, i.e.

$$
\begin{cases}u^{\beta} \geq 0, & (x, \tau) \in \Omega_{T} \\ \partial_{\tau} u^{\beta}-\widetilde{\mathcal{L}} u^{\beta} \geq \beta e^{x}-c(1-\gamma), & (x, \tau) \in \Omega_{T} \\ u^{\beta}\left[u_{\tau}^{\beta}-\widetilde{\mathcal{L}} u^{\beta}-\beta e^{x}+c(1-\gamma)\right]=0, & (x, \tau) \in \Omega_{T} \\ u^{\beta}(x, 0)=\left(e^{x}-P\right)^{+}, & x \in R,\end{cases}
$$

where $\Omega_{T}=R \times(0, T]$ and

$$
\widetilde{\mathcal{L}} u=\frac{\sigma^{2}}{2} \partial_{x x} u+\left(r-\beta-\frac{\sigma^{2}}{2}\right) \partial_{x} u-r u
$$

when $\beta e^{x} \geq c$, it is optimal to pay all the residual cash flows as dividends. When $\beta e^{x}<c$, it is optimal to pay no dividends. This is consistent with the result of Fan and Sundaresan in [8]. 
In the following, we devote the analysis to the behavior of the free boundary (i.e. optimal bankruptcy boundary).

Denote

$$
\begin{array}{ll}
\mathbf{C R}=\{(x, \tau): u(x, \tau)>0\}, & \text { the continuation region, } \\
\mathbf{B R}=\{(x, \tau): u(x, \tau)=0\}, & \text { the bankruptcy region. }
\end{array}
$$

From (3.8), we can see that the region $\mathbf{B R} \subset\left\{x \leq \ln \frac{c}{\beta}\right\}$.

\section{Free boundary in the case of $c(1-\gamma)-r P \leq 0$}

In this section, we aim to characterize the regularities of the free boundary between the region $\mathbf{C R}$ and the region BR. It is worthwhile pointing out that, without consideration of the dividend policy, Han et al. in [10] deduced that $\partial_{x} u \geq 0$, which plays an important role in the analysis of the regularities of the free boundary. But it is not true in this paper. Fortunately, we have the following lemma, which enables us to define the free boundary another way.

Lemma 4.1 If $c(1-\gamma)-r P \leq 0$, then the solution to problem (2.10) satisfies

$$
\begin{aligned}
& u \geq\left(e^{x}-P\right)^{+}, \\
& \partial_{\tau} u \geq 0 .
\end{aligned}
$$

Proof We denote $V=e^{x}-P$, then we have

$$
\begin{cases}\partial_{\tau} V-\mathcal{L} V-g(x) e^{x}+c(1-\gamma)=-r P+c(1-\gamma) \leq 0, & (x, \tau) \in \Omega_{T}, \\ V(x, 0)=e^{x}-P, & x \in R .\end{cases}
$$

In view of the estimation (3.6), we can apply the comparison principle to the system $(2.10)_{2}$, $(2.10)_{4}$, and (4.3), then we have

$$
u \geq e^{x}-P .
$$

Combining estimation (3.6), we have

$$
u \geq\left(e^{x}-P\right)^{+} .
$$

On the other hand, for any small $\delta>0$, denote $\hat{u}(x, \tau)=u(x, \tau+\delta)$, then by $(2.10)$,

$$
\begin{cases}\hat{u}(x, \tau) \geq 0, & (x, \tau) \in \Omega_{T-\delta}, \\ \partial_{\tau} \hat{u}(x, \tau)-\mathcal{L} \hat{u}(x, \tau) \geq g(x) e^{x}-c(1-\gamma), & (x, \tau) \in \Omega_{T-\delta}, \\ \hat{u}(x, \tau)\left[\partial_{\tau} \hat{u}(x, \tau)-\mathcal{L} \hat{u}(x, \tau)-g(x) e^{x}+c(1-\gamma)\right]=0, & (x, \tau) \in \Omega_{T-\delta}, \\ \hat{u}(x, 0)=u(x, \delta) \geq\left(e^{x}-P\right)^{+}=u(x, 0), & x \in R,\end{cases}
$$

where $\Omega_{T-\delta}=R \times(0, T-\delta]$. 
Applying the comparison principle with respect to the initial value of variational inequality (see [12]), we obtain

$$
u(x, \tau+\delta)=\hat{u}(x, \tau) \geq u(x, \tau), \quad(x, \tau) \in R \times(0, T-\delta] .
$$

So, we have

$$
\partial_{\tau} u \geq 0, \quad(x, \tau) \in \Omega_{T}
$$

By (4.2), $u$ is monotonically increasing with respect to $\tau$, so we can define the free boundary

$$
F(x)=\sup \{\tau: u(x, \tau)=0\}, \quad x \in R
$$

It is clear that

$$
\begin{aligned}
& \mathbf{B R}=\{(x, \tau): 0<\tau \leq F(x)\}, \\
& \mathbf{C R}=\{(x, \tau): F(x)<\tau \leq T\} .
\end{aligned}
$$

Lemma 4.2 If $c(1-\gamma)-r P \leq 0$, the free boundary $F(x)$ is decreasing, moreover, $F(x)$ is strictly decreasing and continuous in the region $\{x: 0<F(x)<T\}$.

Proof We divide the proof into four steps.

Step 1: We will deduce that if $F(x)>0$, then $x \leq \min \left\{\ln \frac{c(1-\gamma)}{\beta}, \ln P\right\}$.

From the initial condition $u(x, 0)=\left(e^{x}-P\right)^{+}$and the estimation (4.2), we can conclude that $u>0$ when $x>\ln P$. On the other hand, when $(x, \tau) \in \mathbf{B R}, u=0$, then from estimation (3.8) we have $x \leq \ln \frac{c}{\beta}$ and $g(x)=\beta$, combining with $(2.10)_{2}$, we deduce that $x \leq \ln \frac{c(1-\gamma)}{\beta}$. From the free boundary definition of (4.4) we have the conclusion.

Step 2: We will prove that $F(x)$ is decreasing in $\{x: F(x)>0\}$.

For any $F\left(x_{0}\right)>0$, from step 1 we know that $x_{0} \leq \min \left\{\ln \frac{c(1-\gamma)}{\beta}, \ln P\right\}$. We define

$$
\widetilde{u}(x, \tau)= \begin{cases}u(x, \tau), & (x, \tau) \in\left[x_{0},+\infty\right) \times\left[0, F\left(x_{0}\right)\right] \\ 0, & (x, \tau) \in\left(-\infty, x_{0}\right) \times\left[0, F\left(x_{0}\right)\right]\end{cases}
$$

We will prove that $\widetilde{u}(x, \tau)$ is also the solution of problem (2.10). Since $x_{0} \leq \ln P$, it is easy to prove that $\widetilde{u}(x, \tau)$ satisfies $(2.10)_{1}$ and $(2.10)_{4}$. When $x \leq x_{0} \leq \ln \frac{c(1-\gamma)}{\beta}, \widetilde{u}(x, \tau)=0$,

$$
\partial_{\tau} \widetilde{u}(x, \tau)-\mathcal{L} \widetilde{u}(x, \tau)-g(x) e^{x}+c(1-\gamma)=-\beta e^{x}+c(1-\gamma) \geq 0 .
$$

Thus we can conclude $\widetilde{u}(x, \tau)$ is solution of $(2.10)$ and $u(x, \tau)=\widetilde{u}(x, \tau)$ when $(x, \tau) \in$ $(-\infty,+\infty) \times\left[0, F\left(x_{0}\right)\right]$ for the uniqueness of the solution. So for any point $x \leq x_{0}$, we have $u\left(x, F\left(x_{0}\right)\right)=0$, combining the estimation (4.2) and free boundary definition (4.4) we conclude that $F(x) \geq F\left(x_{0}\right)$, i.e. the free boundary $F(x)$ is decreasing in $\{x: F(x)>0\}$.

Step 3: We infer $F(x)$ is strictly decreasing in $\{x: 0<F(x)<T\}$. 
Otherwise, there exist $x_{1}, x_{2},\left(x_{1}<x_{2} \leq \min \left\{\ln \frac{c(1-\gamma)}{\beta}, \ln P\right\}\right)$, such that $0<F(x)=F\left(x_{1}\right)<$ $T, x \in\left[x_{1}, x_{2}\right]$. There exists a domain $\left(x_{1}, x_{2}\right) \times\left(F\left(x_{1}\right), T\right]$, such that

$$
\begin{cases}\partial_{\tau} u-\mathcal{L} u=\beta e^{x}-c(1-\gamma), & (x, \tau) \in\left(x_{1}, x_{2}\right) \times\left(F\left(x_{1}\right), T\right], \\ u\left(x, F\left(x_{1}\right)\right)=0, & x \in\left(x_{1}, x_{2}\right) .\end{cases}
$$

Then we have $\partial_{\tau} u\left(x, F\left(x_{1}\right)\right)=\beta e^{x}-c(1-\gamma)+\mathcal{L} u\left(x, F\left(x_{1}\right)\right)=\beta e^{x}-c(1-\gamma)<0$ for any $x \in$ $\left(x_{1}, x_{2}\right)$, which contradicts (4.2). Hence $F(x)$ is strictly decreasing in $\{x: 0<F(x)<T\}$.

Step 4: We conclude $F(x)$ is continuous in $\{x: 0<F(x)<T\}$.

Otherwise there exists a point $x_{0}$ such that $\lim _{x \rightarrow x_{0}^{+}} F(x)=\tau_{1}<\tau_{2}=\lim _{x \rightarrow x_{0}^{-}} F(x)$ and

$$
\begin{cases}\partial_{\tau} u-\mathcal{L} u=\beta e^{x}-c(1-\gamma), & (x, \tau) \in\left(x_{0}, \ln \frac{c}{\beta}\right) \times\left(\tau_{1}, \tau_{2}\right), \\ u\left(x_{0}, \tau\right)=0, & \tau \in\left(\tau_{1}, \tau_{2}\right) .\end{cases}
$$

Then $u \in C^{\infty}\left[x_{0}, \ln \frac{c}{\beta}\right) \times\left(\tau_{1}, \tau_{2}\right)$. Denote $w=\partial_{\tau} u$, then $w$ satisfies

$$
\begin{cases}\partial_{\tau} w-\mathcal{L} w=0, & (x, \tau) \in\left(x_{0}, \ln \frac{c}{\beta}\right) \times\left(\tau_{1}, \tau_{2}\right), \\ w\left(x_{0}, \tau\right)=0, & \tau \in\left(\tau_{1}, \tau_{2}\right) .\end{cases}
$$

Since $w=\partial_{\tau} u \geq 0, w$ reaches its non-positive minimums at $x=x_{0}$; applying the maximum principle we have $\partial_{x} w\left(x_{0}, \tau\right)>0$ for any $\tau \in\left(\tau_{1}, \tau_{2}\right)$. On the other hand, $u(x, \tau)=0,(x, \tau) \in$ $\left(x_{0}-1, x_{0}\right] \times\left(\tau_{1}, \tau_{2}\right)$, moreover, $\partial_{x} u \in C\left(\Omega_{T}\right)$, thus we can deduce that $\partial_{x} u\left(x_{0}, \tau\right)=0$ for any $\tau \in\left(\tau_{1}, \tau_{2}\right)$. So, $\partial_{x} w\left(x_{0}, \tau\right)=\partial_{\tau x} u\left(x_{0}, \tau\right)=0$ for any $\tau \in\left(\tau_{1}, \tau_{2}\right)$, thus we get a contradiction. Hence, $F(x)$ is continuous in $\{x: 0<F(x)<T\}$.

Since the free boundary $\tau=F(x)(x \in R)$ is strictly decreasing in $(0, T)$, there exists an inverse function $x=h(\tau)$, and we define the free boundary

$$
h(\tau)=\{x: \tau=F(x)\}, \quad \tau \in(0, T) .
$$

Theorem 4.1 If $c(1-\gamma)-r P \leq 0$, the free boundary (see Figure 3$) h(\tau) \in C[0, T] \cap C^{\infty}(0, T]$ is strictly decreasing with

$$
h(0)=\lim _{\tau \rightarrow 0^{+}} h(\tau)=\min \left\{\ln P, \ln \frac{c(1-\gamma)}{\beta}\right\},
$$

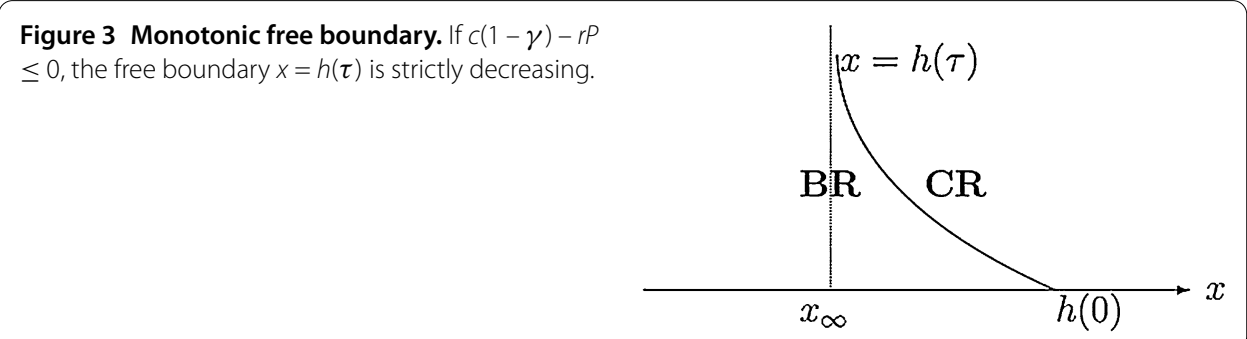


moreover,

$$
x_{\infty} \leq h(\tau) \leq \min \left\{\ln P, \ln \frac{c(1-\gamma)}{\beta}\right\},
$$

where, from Fan and Sundaresan [8], $x_{\infty}$ is the optimal bankruptcy point for perpetual debt and

$$
x_{\infty}=\ln \left(\frac{-\alpha}{1-\alpha} \frac{c(1-\gamma)}{r}\right),
$$

where $\alpha$ is a negative root of the equation

$$
\frac{1}{2} \sigma^{2} x^{2}+\left(r-\delta-\frac{1}{2} \sigma^{2}\right) x-r=0
$$

Proof Since the free boundary $\tau=F(x)$ is strictly decreasing, its inverse function $h(\tau)$ is continuous and strictly decreasing.

Now we prove $h(0)=\lim _{\tau \rightarrow 0^{+}} h(\tau)=\min \left\{\ln P, \ln \frac{c(1-\gamma)}{\beta}\right\}$. From estimation (3.8), we know $h(\tau) \leq \ln \frac{c}{\beta}$, combining its monotonicity we conclude the limitation exists. In the same way as in Lemma 4.2, we can prove the conclusion.

Because $h(\tau)$ is decreasing in $[0, T]$, we can deduce that $x_{\infty}=\lim _{\tau \rightarrow+\infty} h(\tau) \leq h(\tau) \leq$ $h(0)$ for any $\tau \in(0, T]$.

At last, we prove $h(\tau) \in C^{\infty}(0, T]$. Since $\partial_{\tau} u \geq 0$, using the method developed by Friedman [16], it is not difficult to prove $h(\tau) \in C^{0,1}(0, T]$. At this point we can use the result of the Stefan problem [17] to find that $h(\tau) \in C^{\infty}(0, T]$.

\section{Free boundary in the case of $c(1-\gamma)-r P>0$}

In this section, we aim to investigate the behaviors of the free boundary in the case of $c(1-\gamma)-r P>0$. However, when $c(1-\gamma)-r P>0$, the inequality $\partial_{\tau} u \geq 0$ is no longer satisfied. A numerical example is presented in Section 6. In [10], Han et al. obtained a similar property to $\partial_{\tau} u \geq 0$ by making the transformation, which is no longer true since the function $g(x)$ is a piecewise function. In addition, $g(x)$ is discontinuous on the point $x=\ln \frac{c}{\beta}$, which causes it to be difficult to prove that $\partial_{x} u \geq 0$. So we cannot prove the free boundary is differentiable. Fortunately, by establishing linkages between problem (2.10) and (3.12), we deduce that the free boundary is bounded and no longer monotonic in some cases.

For comparison, let us first recall the results in [10] where the authors do not take the optimal dividend policy into account. In the absence of a dividend policy, the counterpart of (2.10) becomes (3.12) (see [10] and we assume $\eta \alpha=0)$. Problem (3.12) has a free boundary, denoted by $\widetilde{h}(\tau)$, such that

$$
\widetilde{h}(\tau)=\sup \left\{x \in R, u^{\beta}(x, \tau)=0\right\}, \quad \tau \in(0, T] .
$$

Han et al. [10] completely characterized the behaviors of $\widetilde{h}(\tau)$, which is summarized as follows. 
Lemma 5.1 In the case of $c(1-\gamma)-r P>0$, the free boundary $\widetilde{h}(\tau) \in C[0, T] \cap C^{\infty}(0, T]$ and

$$
\ln \left(-\frac{P \alpha_{1}}{1-\alpha_{1}}\right) \leq \widetilde{h}(\tau) \leq \ln \frac{c(1-\gamma)}{\beta}
$$

where $\alpha_{1}$ is the negative root of the algebraic equation

$$
\frac{1}{2} \sigma^{2} x^{2}+\left(r-\beta-\frac{1}{2} \sigma^{2}\right) x-r=0
$$

Moreover,

$$
\widetilde{h}(0):=\lim _{\tau \rightarrow 0^{+}} \tilde{h}(\tau)=\min \left\{\ln P, \ln \frac{c(1-\gamma)}{\beta}\right\} .
$$

Now we define free boundary of problem (2.10) by

$$
h(\tau)=\max \{x \mid u(y, \tau)=0 \text {, for } y \leq x\} .
$$

Theorem 5.1 If $c(1-\gamma)-r P>0$, the free boundary $h(\tau)$ is bounded and starts with

$$
h(0)=\min \left\{\ln P, \ln \frac{c(1-\gamma)}{\beta}\right\}
$$

moreover,

$$
\ln \left(-\frac{P \alpha_{1}}{1-\alpha_{1}}\right) \leq \widetilde{h}(\tau) \leq h(\tau) \leq \ln \frac{c(1-\gamma)}{\beta},
$$

where $\widetilde{h}(\tau)$ is defined by (5.1) and $\alpha_{1}$ is a negative root of (5.3).

Proof When $(x, \tau) \in \mathbf{B R}, u(x, \tau)=0$, combining the estimation (3.8), we deduce that $x \leq$ $\ln \frac{c}{\beta}$, then $g(x)=\beta$. From $(2.10)_{2}$, we have $x \leq \ln \frac{c(1-\gamma)}{\beta}$. Hence, we obtain the right hand side of inequality (5.4).

On the other hand, from [10], we know that when $x \leq \widetilde{h}(\tau)$ (where $\widetilde{h}(\tau)$ is the optimal reorganization boundary of problem (3.12)), $u^{\beta}(x, \tau)=0$. From (3.11), we have $u(x, \tau) \leq$ $u^{\beta}(x, \tau)$, noticing that $u(x, \tau) \geq 0$, then we can infer that $u(x, \tau)=0$ for all $x \leq \widetilde{h}(\tau)$. Thus we conclude that $\widetilde{h}(\tau) \leq h(\tau)$. By Lemma $5.1, \ln \left(-\frac{P \alpha_{1}}{1-\alpha_{1}}\right) \leq \widetilde{h}(\tau)$, so we obtain inequality (5.4).

Next, we prove $h(0)=\min \left\{\ln P, \ln \frac{c(1-\gamma)}{\beta}\right\}$. Since $u(x, \tau) \in C\left(\bar{\Omega}_{T}\right)$ and $u(x, 0)=\left(e^{x}-P\right)^{+}$, we have $h(0) \leq \ln P$. Combining (5.4), we have $h(0) \leq \min \left\{\ln P, \ln \frac{c(1-\gamma)}{\beta}\right\}$. If $h(0)<$ $\min \left\{\ln P, \ln \frac{c(1-\gamma)}{\beta}\right\}$, there exists a domain $(h(0), h(0)+\epsilon) \times(0, t) \subset(h(0), \min \{\ln P$, $\left.\left.\ln \frac{c(1-\gamma)}{\beta}\right\}\right) \times(0, T]$, such that

$$
\begin{cases}\partial_{\tau} u-\mathcal{L} u+\beta \partial_{x} u=\beta e^{x}-c(1-\gamma), & (x, \tau) \in(h(0), h(0)+\epsilon) \times(0, t), \\ u(x, 0)=0, & x \in(h(0), h(0)+\epsilon) .\end{cases}
$$




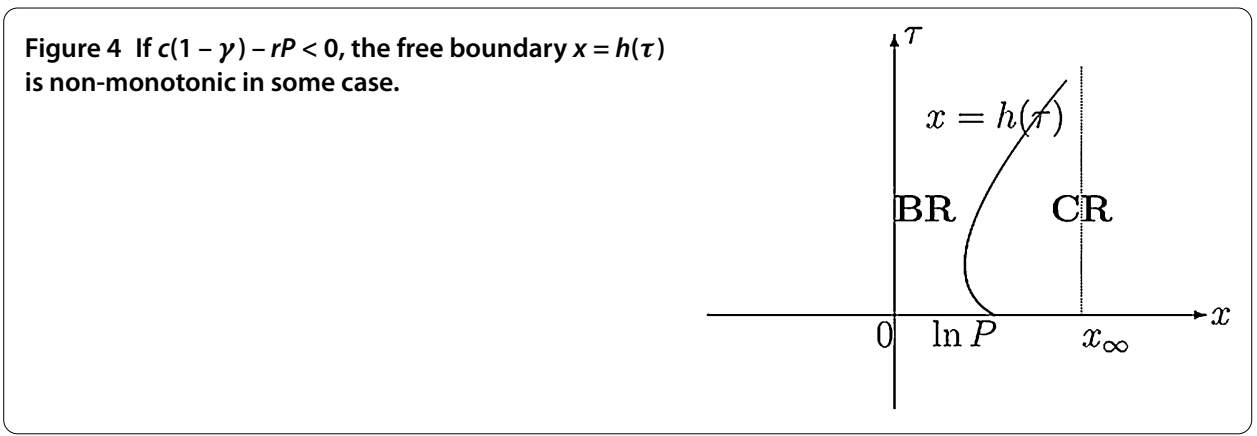

Then we have $\partial_{\tau} u(x, 0)=\beta e^{x}-c(1-\gamma)+\mathcal{L} u(x, 0)-\beta \partial_{x} u(x, 0)=\beta e^{x}-c(1-\gamma)<0$ for any $x \in(h(0), h(0)+\epsilon)$, which contradicts $u(x, \tau) \geq 0$ for all $0<\tau \leq T$. Hence $h(0)=$ $\min \left\{\ln P, \ln \frac{c(1-\gamma)}{\beta}\right\}$.

Next, we prove that the free boundary is not monotonic in some cases.

Theorem 5.2 In the case of $c(1-\gamma)-r P>0$, the free boundary $h(\tau)$ (see Figure 4$)$ is nonmonotonic in the interval $[0,+\infty)$ if

$$
\ln P<x_{\infty}
$$

where $x_{\infty}$ is defined by (4.5).

Proof Since $\alpha$ is the negative root of (4.6), we can infer that $\ln \left[\frac{-\alpha}{1-\alpha} \cdot \frac{c(1-\gamma)}{r}\right]<\ln \frac{c(1-\gamma)}{\beta}$, thus if $\ln P<x_{\infty}=\ln \left[\frac{-\alpha}{1-\alpha} \cdot \frac{c(1-\gamma)}{r}\right]$, we deduce that $h(0)=\min \left\{\ln P, \ln \frac{c(1-\gamma)}{\beta}\right\}=\ln P$.

First, we prove that there exists a positive $\tau_{0}$ such that $h(\tau)<h(0)$ for any $\tau \leq \tau_{0}$.

We set

$$
y=x-\ln P, \quad w(y, \tau)=\frac{1}{P} u(x, \tau) .
$$

Recall (2.10), it is not difficult to find $w$ is governed by

$$
\begin{cases}w \geq 0, & (y, \tau) \in R \times(0, T], \\ \partial_{\tau} w-\mathcal{L} w+g(y+\ln P)\left(\partial_{y} w-e^{y}\right) \geq-\frac{c(1-\gamma)}{P} & (y, \tau) \in R \times(0, T], \\ w\left(\partial_{\tau} w-\mathcal{L} w+g(y+\ln P)\left(\partial_{y} w-e^{y}\right)+\frac{c(1-\gamma)}{P}\right)=0, & (y, \tau) \in R \times(0, T], \\ w(y, 0)=\left(e^{y}-1\right)^{+}, & y \in R,\end{cases}
$$

where

$$
\mathcal{L} w=\frac{\sigma^{2}}{2} \partial_{y y} w+\left(r-\frac{\sigma^{2}}{2}\right) \partial_{y} w-r w .
$$

From (3.7), we deduce that $\partial_{y} w(y, \tau) \leq e^{y}$ for any $(y, \tau) \in R \times(0, T]$. We construct a subsolution $\widetilde{w}$ to problem (5.5), which satisfies

$$
\begin{cases}\partial_{\tau} \widetilde{w}-\mathcal{L} \widetilde{w}=-\frac{c(1-\gamma)}{P}, & (y, \tau) \in R \times(0, T] \\ \widetilde{w}(y, 0)=\left(e^{y}-1\right)^{+}, & y \in R .\end{cases}
$$


It is clear that $w \geq \widetilde{w}$ by the comparison principle. If we can prove that there exists a positive $\tau_{0}$, such that $\widetilde{w}(0, \tau)>0$ for any $0<\tau<\tau_{0}$, then $w(0, \tau)>0$.

We define $\widetilde{w}(y, \tau)=v(y, \tau)+\frac{c(1-\gamma)}{r P}\left(e^{-r \tau}-1\right)$, where $v$ satisfies

$$
\begin{cases}\partial_{\tau} v-\mathcal{L} v=0, & (y, \tau) \in R \times(0, T] \\ v(y, 0)=\left(e^{y}-1\right)^{+}, & y \in R\end{cases}
$$

In fact, $v$ is the premium of the European call option with strike price 1 and dividend 0 . We can obtain the solution of (5.7) (see [18])

$$
v(y, \tau)=e^{y} N\left(\hat{d}_{1}\right)-e^{-r \tau} N\left(\hat{d}_{2}\right),
$$

where

$$
N(x)=\frac{1}{\sqrt{2 \pi}} \int_{-\infty}^{x} e^{-t^{2} / 2} d t, \quad \hat{d}_{1}=\frac{y+\left(r+\sigma^{2} / 2\right) \tau}{\sigma \sqrt{\tau}}, \quad \hat{d}_{2}=\frac{y+\left(r-\sigma^{2} / 2\right) \tau}{\sigma \sqrt{\tau}} .
$$

It is not difficult to check that

$$
\partial_{\tau} v(0, \tau)=n\left(d_{1}\right) \frac{r+\sigma^{2} / 2}{2 \sigma \sqrt{\tau}}+r e^{-r \tau} N\left(d_{2}\right)-e^{-r \tau} n\left(d_{2}\right) \frac{r-\sigma^{2} / 2}{2 \sigma \sqrt{\tau}} \geq n\left(d_{1}\right) \frac{\sigma}{2 \sqrt{\tau}},
$$

where

$$
n(x)=\frac{e^{-x^{2} / 2}}{\sqrt{2 \pi}}, \quad d_{1}=\frac{\left(r+\sigma^{2} / 2\right) \sqrt{\tau}}{\sigma}, \quad d_{2}=\frac{\left(r-\sigma^{2} / 2\right) \sqrt{\tau}}{\sigma} .
$$

(The last inequality is obtained from the fact $n\left(d_{1}\right)=e^{-r \tau} n\left(d_{2}\right)$.)

Then we have

$$
\partial_{\tau} \nu(0, \tau) \rightarrow+\infty, \quad \text { as } \tau \rightarrow 0^{+} .
$$

So

$$
\partial_{\tau} \widetilde{w}(0, \tau) \rightarrow+\infty, \quad \text { as } \tau \rightarrow 0^{+}
$$

Since $\widetilde{w}(0,0)=0$ and $\widetilde{w} \in C(R \times[0, T])$, there exists a positive $\tau_{0}$ such that

$$
\widetilde{w}(0, \tau)>0, \quad \tau \leq \tau_{0} .
$$

Thus, there exists a positive $\tau_{0}$, such that $h(\tau)<h(0)$ for any $\tau \leq \tau_{0}$; combining the condition $h(0)<x_{\infty}$ and $\lim _{\tau \rightarrow+\infty} h(\tau)=x_{\infty}$, we know that the free boundary $h(\tau)$ is not monotonic in the interval $[0,+\infty)$.

\section{Numerical results}

We start from problem (2.10), which can be rewritten as

$$
\begin{cases}\min \left\{\partial_{\tau} u-\mathcal{L} u+g(x)\left(\partial_{x} u-e^{x}\right)+c(1-\gamma), u\right\}=0, & (x, \tau) \in \Omega_{T}, \\ u(x, 0)=\left(e^{x}-P\right)^{+}, & x \in R,\end{cases}
$$


where $\Omega_{T}=R \times(0, T]$ and

$$
\mathcal{L} u=\frac{\sigma^{2}}{2} \partial_{x x} u+\left(r-\frac{\sigma^{2}}{2}\right) \partial_{x} u-r u
$$

and

$$
g(x)= \begin{cases}\beta, & x \leq \ln \frac{c}{\beta} \\ \delta, & x>\ln \frac{c}{\beta}\end{cases}
$$

Given mesh size $\Delta x, \Delta \tau>0, u_{j}^{n}=u(j \Delta x, n \Delta \tau)$ represents the value of the numerical approximation at $(j \Delta x, n \Delta \tau)$, then (6.1) becomes the following difference equation:

$$
\left\{\begin{aligned}
\min & \left\{\frac{u_{j}^{n}-u_{j}^{n-1}}{\Delta \tau}-\frac{\sigma^{2}}{2} \frac{u_{j+1}^{n-1}-2 u_{j}^{n-1}+u_{j-1}^{n-1}}{\Delta x^{2}}-\left(r-g(j \Delta x)-\frac{\sigma^{2}}{2}\right) \frac{u_{j+1}^{n-1}-u_{j-1}^{n-1}}{2 \Delta x}\right. \\
& \left.+r u_{j}^{n}-g(j \Delta x) e^{j \Delta x}+c(1-\gamma), u_{j}^{n}\right\}=0 \\
u_{j}^{0} & =\left(e^{j \Delta x}-P\right)^{+} .
\end{aligned}\right.
$$

Denote $\omega=\frac{\sigma^{2} \Delta \tau}{\Delta x^{2}}, a=\frac{\omega}{2}+\left(r-g(j \Delta x)-\frac{\sigma^{2}}{2}\right) \frac{\Delta \tau}{2 \Delta x}, b=\omega-a$; from (6.2) we calculate that

$$
\left\{\begin{array}{l}
\min \left\{(1+r \Delta \tau) u_{j}^{n}-(1-\omega) u_{j}^{n-1}-a u_{j+1}^{n-1}-b u_{j-1}^{n-1}-g(j \Delta x) e^{j \Delta x} \Delta \tau\right. \\
\left.\quad+c(1-\gamma) \Delta \tau, u_{j}^{n}\right\}=0, \\
u_{j}^{0}=\left(e^{j \Delta x}-P\right)^{+} .
\end{array}\right.
$$

Choosing $\omega=1$, we have

$$
\left\{\begin{array}{l}
u_{j}^{n}=\max \left\{\frac{1}{1+r \Delta \tau}\left[a u_{j+1}^{n-1}+b u_{j-1}^{n-1}+, g(j \Delta x) e^{j \Delta x} \Delta \tau-c(1-\gamma) \Delta \tau\right], 0\right\} \\
u_{j}^{0}=\left(e^{j \Delta x}-P\right)^{+}
\end{array}\right.
$$

where $j \in Z, 1 \leq n \leq N, N=\frac{T}{\Delta \tau}$, then we get Figures 5-10.

Remark 6.1 The numerical results in Figure 5 and Figure 6 reveal that the bankrupt boundary $h(\tau)$ is decreasing with respect to the volatility $\sigma$. The financial meaning is this: when the volatility increases, the firm need not bankrupt at once, because of a possibility of increasing for $V$ due to the big volatility.

Remark 6.2 The numerical results in Figure 7 and Figure 8 show that the bankrupt boundary is decreasing with respect to the risk-free rate $r$. The financial explanation is very simple. The increase of the risk-free rate $r$ results in the increase of $V$. Hence $E(v, t)$ increases, then bankruptcy is behind of schedule.

Remark 6.3 Figure 9 and Figure 10 show that, as the payout ratio $\delta$ increases from $\beta(1-\gamma)$ to $\beta$, the bankrupt boundary is decreasing. The financial explanation is very simple. If the residual cash flows are invested back as retained earning, they become accessible by the debt holders upon bankruptcy. It would be optimal for the equity holders to pay all the cash flows available as divided. 


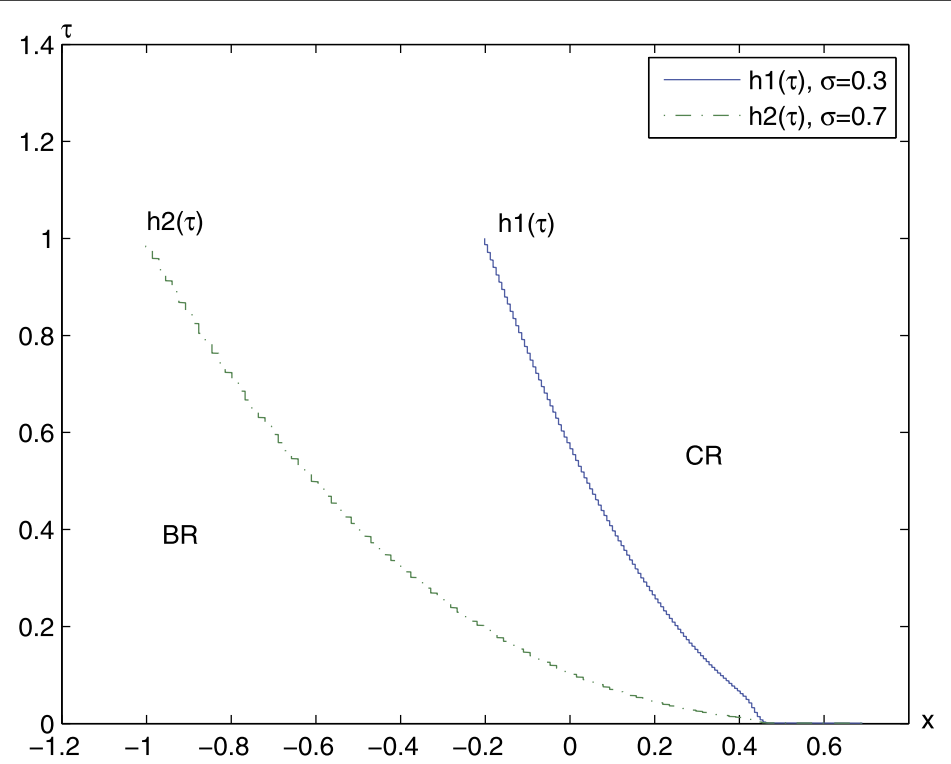

Figure 5 The free boundary when $c(1-\gamma)-r P \leq 0$ with different volatility. Plot of the optimal bankruptcy boundary $h(\tau)$ as the function of time $\tau$ when $c(1-\gamma)-r P \leq 0$. The parameter values used in the calculations are $T=1, N=2,000, r=0.3, \beta=0.02, \delta=0.01, c=0.04, \gamma=0.2, P=2 ; h 1(\tau)$ and $h 2(\tau)$ are the free boundaries when $\sigma_{1}=0.3$ and $\sigma_{2}=0.7$, respectively. The numerical result (see Figure 5 ) shows that the optimal bankruptcy boundary is decreasing not only with $\tau$, which coincides with Theorem 4.1 , but also with volatility $\sigma$.

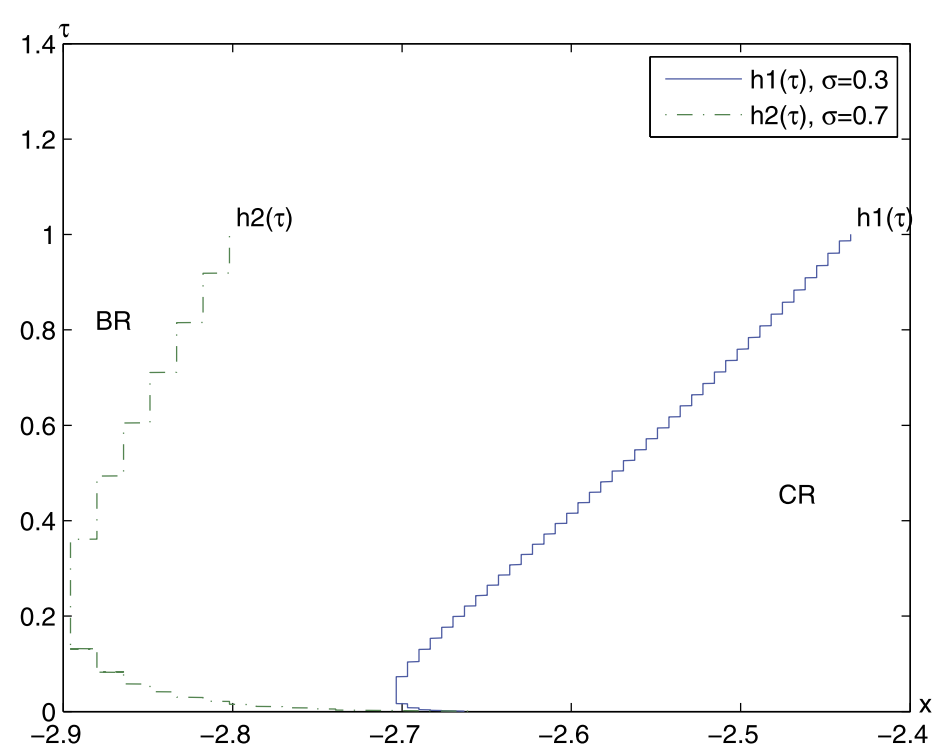

Figure 6 The free boundary when $c(1-\gamma)-r P>0$ with different volatility. Plot of the optimal bankruptcy boundary $h(\tau)$ as the function of time $\tau$ when $c(1-\gamma)-r P>0$. The parameter values used in the calculations are $T=1, N=2,000, r=0.03, \beta=0.02, c=0.04, \delta=0.01, \gamma=0.2, P=0.07 ; h 1(\tau)$ and $h 2(\tau)$ are the free boundaries when $\sigma_{1}=0.3$ and $\sigma_{2}=0.7$, respectively. The numerical result (see Figure 6 ) shows that the optimal bankrupt boundary $h(\tau)$ is not monotonic with $\tau$, which coincides with Theorem 5.2 , at the same time, the numerical result also reveals the bankruptcy boundary $h(\tau)$ is decreasing with respect to volatility $\sigma$. 


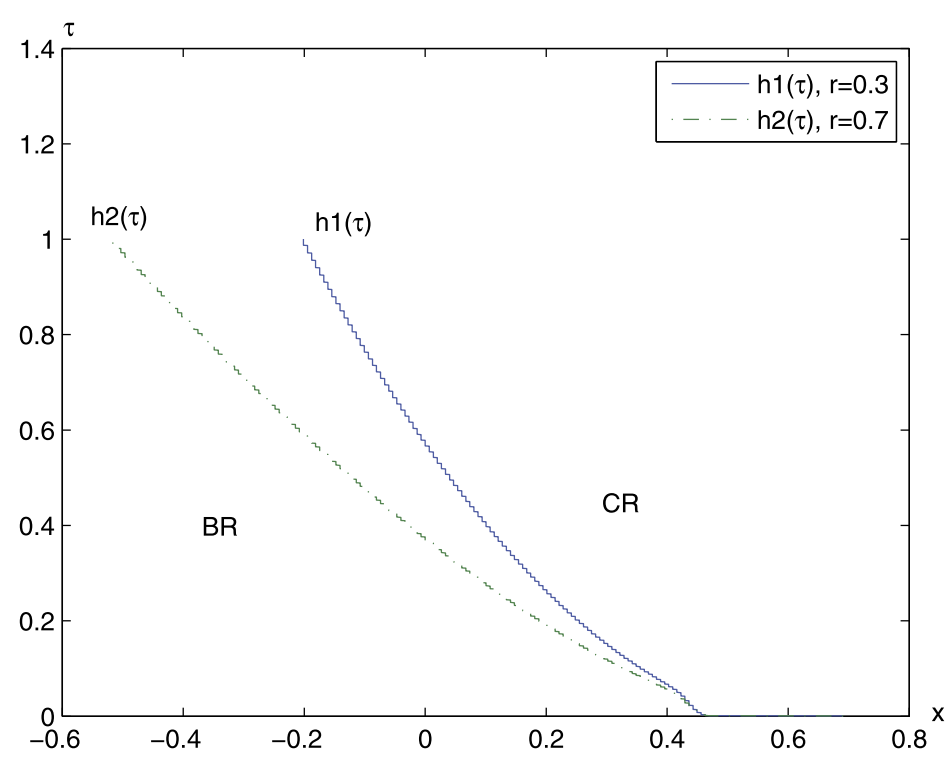

Figure 7 The free boundary when $c(1-\gamma)-r P \leq 0$ with different risk-free rate. Plot of the optimal bankruptcy boundary $h(\tau)$ as the function of time $\tau$ when $c(1-\gamma)-r P \leq 0$. The parameter values used in the calculations are $T=1, N=2,000, \sigma=0.3, \beta=0.02, \delta=0.01, c=0.04, \gamma=0.2, P=2 ; h 1(\tau)$ and $h 2(\tau)$ are the free boundaries when $r_{1}=0.3$ and $r_{2}=0.7$, respectively. The numerical result (see Figure 7) shows that the optimal bankruptcy boundary $h(\tau)$ is decreasing not only with $\tau$, which coincides with Theorem 4.1, but also with risk-free rate $r$.



Figure 8 The free boundary when $c(1-\gamma)-r P>0$ with different risk-free rate. Plot of the optimal bankruptcy boundary $h(\tau)$ as the function of time $\tau$ when $c(1-\gamma)-r P>0$. The parameter values used in the calculations are $T=1, N=2,000, \sigma=0.3, \beta=0.02, c=0.04, \delta=0.01, \gamma=0.2, P=0.07 ; h 1(\tau)$ and $h 2(\tau)$ are the free boundaries when $r_{1}=0.03$ and $r_{2}=0.07$, respectively. The numerical result (see Figure 8 ) shows that the optimal bankruptcy boundary $h(\tau)$ is not monotonic with $\tau$, which coincides with Theorem 5.2 , at the same time, the numerical result also reveals the bankruptcy boundary $h(\tau)$ is decreasing with respect to risk-free rate $r$. 


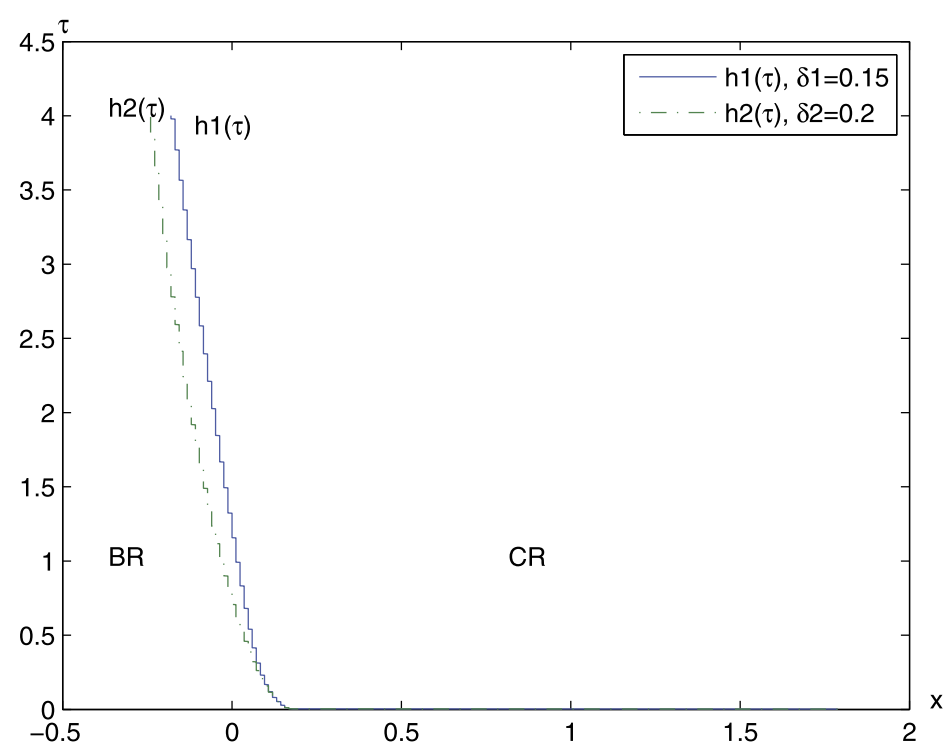

Figure 9 The free boundary when $c(1-\gamma)-r P \leq 0$ with different aggregate payout ratio. Plot of the optimal bankruptcy boundary $h(\tau)$ as the function of time $\tau$ when $c(1-\gamma)-r P \leq 0$. The parameter values used in the calculations are $T=4, N=2,500, \sigma=0.3, r=0.3, \beta=0.2, c=0.3, \gamma=0.2, P=6 ; h 1(\tau)$ and $h 2(\tau)$ are the free boundaries when $\delta_{1}=0.15$ and $\delta_{2}=0.2$, respectively. The numerical result (see Figure 9) shows that the optimal bankruptcy boundary $h(\tau)$ is decreasing not only with $\tau$, which coincides with Theorem 4.1 , but also with aggregate payout ratio $\delta$.

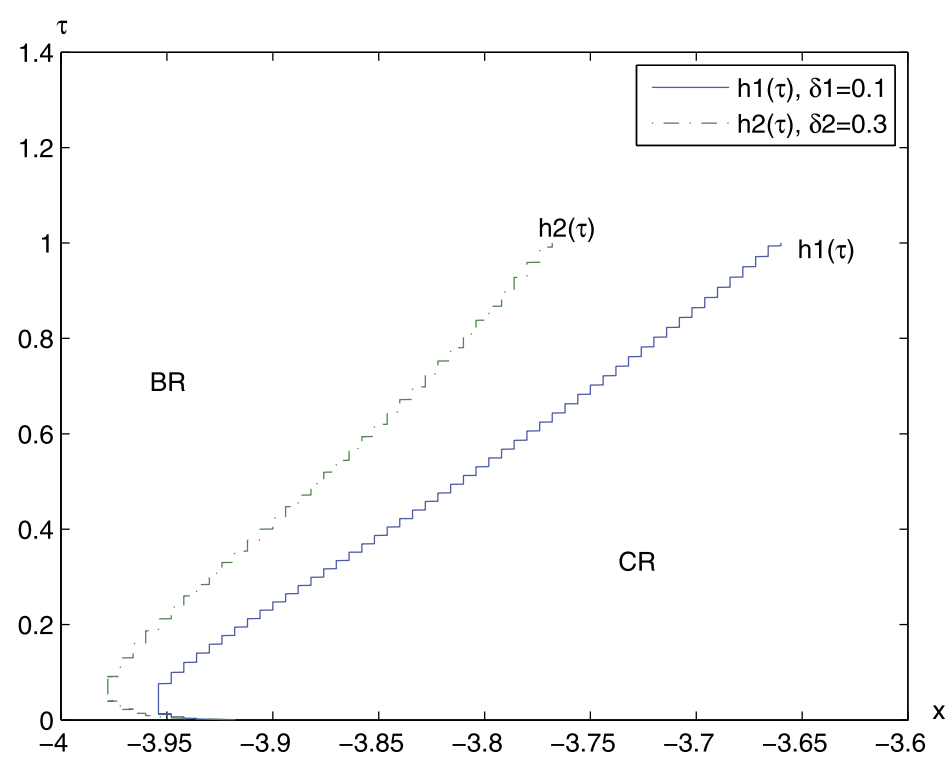

Figure 10 The free boundary when $c(1-\gamma)-r P>0$ with different aggregate payout ratio. Plot of the optimal bankruptcy boundary $h(\tau)$ as the function of time $\tau$ when $c(1-\gamma)-r P>0$. The parameter values used in the calculations are $T=1, N=2,500, \sigma=0.3, r=0.01, \beta=0.8, c=0.01, \gamma=0.001, P=0.02 ; h 1(\tau)$ and $h 2(\tau)$ are the free boundaries when $\delta_{1}=0.1$ and $\delta_{2}=0.3$, respectively. The numerical result (see Figure 10) shows that the optimal bankruptcy boundary $h(\tau)$ is not monotonic with $\tau$, which coincides with Theorem 5.2, at the same time, the numerical result also reveals the bankruptcy boundary $h(\tau)$ is decreasing with respect to the aggregate payout ratio $\delta$. 


\section{Conclusion}

In this paper, we study the value of equity, optimal bankruptcy and dividend policy in a continuous-time framework with finite time maturity. Most of the previous works either take only an infinite time horizon into consideration or pay out all residual cash flows as dividend.

Mathematically the model of equity value is formulated as a parabolic variational inequality with discontinuous coefficient, or equivalently, a free boundary problem, where the free boundary corresponds to the optimal bankruptcy boundary. We aim to investigate the behaviors of the free boundary and optimal dividend policy.

As we know, the results in this paper are the first integral one for optimal dividends due to the use of the PDE technique. First we rigorously established variational inequality model (2.6) by stochastic analysis. we prove that the solution is increasing with the aggregate payout ratio and obtain the optimal dividend policy. The results are perfect in the case of $c(1-\gamma)-r P \leq 0$. In Section 5 we deduced that in the case of $c(1-\gamma)-r P>0$ the bankruptcy boundary is bounded and we show its loss of monotonicity in some cases. We presented some numerical results and financial interpretations in Section 6.

At time $t$, if the assert value of the firm $v$ is in the continuation region, then the firm should not go bankrupt, and if $v$ is in the bankruptcy region, then the firm should go bankrupt at once.

\section{Competing interests}

The authors declare that they have no competing interests.

\section{Authors' contributions}

The authors read and approved the final manuscript.

\section{Acknowledgements}

The project is supported by National Natural Science Foundation of China (Nos. 11271143, 11371155 and 11326123), University Special Research Fund for Ph.D. Program of China (20124407110001 and 20114407120008) and Foundation for Distinguished Young Talents in High Education of Guangdong (No. 2014KQNCX181).

Received: 7 August 2014 Accepted: 3 February 2015 Published online: 24 February 2015

\section{References}

1. Merton, R: On the pricing of corporate debt: the risk structure of interest rates. J. Finance $29,449-470$ (1974)

2. Black, F, Cox, J: Valuing corporate securities: some effects of bond indenture provisions. J. Finance 31, 351-367 (1976)

3. Leland, H: Corporate debt value, bond covenants, and optimal capital structure. J. Finance 49, 1213-1252 (1994)

4. Anderson, R, Sundaresan, S: The design and valuation of debt contracts. Rev. Financ. Stud. 9, 37-68 (1996)

5. Bruche, M: Creditor coordination, liquidation timing, and debt valuation. J. Financ. Quant. Anal. 46, 1407-1436 (2011)

6. Zhou, C: The term structure of credit spreads with jump risk. J. Bank. Finance 25, 2015-2040 (2001)

7. Leland, H, Toft, K: Optimal capital structure, endogenous bankruptcy, and the term structure of credit spreads. J. Finance 51, 987-1019 (1996)

8. Fan, H, Sundaresan, S: Debt valuation, renegotiation, and optimal dividend policy. Rev. Financ. Stud. 13, 1057-1099 (2000)

9. Gryglewicz, S: A theory of corporate financial decisions with liquidity and solvency concerns. J. Financ. Econ. 99, 365-384 (2011)

10. Han, X, Yi, F, Zhang, J: Debt-equity swap with finite time horizon-variational inequality approach. J. Math. Anal. Appl. 414, 296-318 (2014)

11. Pham, H: Continuous-Time Stochastic Control and Optimization with Financial Applications, pp. 37-58. Springer, Berlin (2009)

12. Friedman, A: Variational Principle and Free Boundary Problems, pp. 79-81. Wiley, New York (1982)

13. Lieberman, G: Second Order Parabolic Differential Equations, pp. 133-135. World Scientific, London (1996)

14. Chen, Y: Parabolic Partial Differential Equations of Second Order, pp. 116-118. Beijing University Press, Beijing (2003)

15. Tao, K: On an Aleksandrov Bakel' man type maximum principle for second order parabolic equations. Commun. Partial Differ. Equ. 10, 543-553 (1985)

16. Friedman, A: Parabolic variational inequalities in one space dimension and smoothness of the free boundary. J. Funct. Anal. 18, 151-176 (1975)

17. Jiang, L: Existence and differentiability of a two-phase Stefan problem for quasilinear parabolic equations. Acta Math. Sin. 15, 481-496 (1965)

18. Jiang, L: Mathematical Modeling and Methods of Option Pricing. World Scientific, Singapore (2005) 